\title{
Erratum to: Case Report: Sodium dichloroacetate (DCA) inhibition of the "Warburg Effect" in a human cancer patient: complete response in non-Hodgkin's lymphoma after disease progression with rituximab-CHOP
}

\author{
Stephen B. Strum • Örn Adalsteinsson • Richard R. Black • \\ Dmitri Segal • Nancy L. Peress • James Waldenfels
}

Published online: 8 May 2013

(C) Springer Science+Business Media New York 2013

Erratum to: J Bioenerg Biomembr (2012)

DOI 10.1007/s10863-012-9496-2

A separate analysis of the same patient was previously presented in a Case Report by Dana F. Flavin: "NonHodgkin's Lymphoma Reversal with Dichloroacetate," Journal of Oncology, vol. 2010, Article ID 414726, 4 pages, 2010. doi: $10.1155 / 2010 / 414726$.

The online version of the original article can be found at http://dx.doi.org/ 10.1007/s10863-012-9496-2.

S. B. Strum $(\bowtie)$

International Strategic Cancer Alliance,

538 Granite Street, Ashland, OR 97520, USA

e-mail: sbstrum@gmail.com

S. B. Strum

American Society of Clinical Oncology,

538 Granite Street, Ashland, OR 97520, USA

S. B. Strum

American Urological Association, 538 Granite Street,

Ashland, OR 97520, USA

Ö. Adalsteinsson

International Strategic Cancer Alliance,

873 E. Baltimore Pike \#333, Kennett Square,

PA 19348, USA

e-mail: ornaa@comcast.net
R. R. Black

Nuclear Medicine and PET Imaging, Radisphere Teleradiology

Group, Beachwood, OH 44022, USA

e-mail: drrickblack@aol.com

D. Segal

Valley Radiology Consultants, Poway, CA 92064, USA

e-mail: dmitrisegal@gmail.com

N. L. Peress

Life Extension Foundation, 5760 S. Scenic Drive,

Sault Sainte Marie, MI 49783, USA

e-mail: nperess@charter.net

J. Waldenfels

Life Extension Foundation, 9114 Steeplebush Court,

Annandale, VA 22003-4051, USA

e-mail: luckyodie2@aol.com 